\title{
L'EXTENSION AFRICAINE EN EUROPE MÉRIDIONALE: LE SOLUTRÉEN
}

\author{
African expansion into Southern Europe: the Solutrean
}

\author{
Marcel Otte ${ }^{1}$ \\ Recibido el 23 de diciembre de 2012. Aceptado el 14 de junio de 2013
}

Résumé. La vaste civilisation atérienne occupait l'ensemble du Sahara actuel, entre le Nil, le Sénégal et le Maghreb durant des dizaines de millénaires. Son outillage est bien connu, et il tend à s'alléger dans ses phases récentes au nord-ouest (Maroc actuel). Désormais, un art rigoureusement paléolithique est connu en Haute-Égypte, daté entre 18 et 23000 BP (Huyge 2013). Ses canons stylistiques sont identiques à ceux des rares sites solutréens ibériques, précisément au moment où les industries bifaciales y pénètrent sous la forme dite de "Solutréen Moyen». Les phases proto-solutréennes, rassemblées sous ce vocable, appartiennent en fait aux variantes gravettiennes, issues d'Europe Centrale et furent mal interprétées. Dans son extension européenne, le Solutréen forme une cassure manifeste, tranchant sur l'histoire de notre continent. Limité à sa partie extrêmement occidentale, il disparaît rapidement dès que les Gravettiens finaux adoptent les modes de vie et les valeurs appropriées vers l'extension et le développement magdalénien.

Mots clés: Solutréen, Afrique, nationalisme, arts plastiques.

Abstract. The vast Aterian civilization occupied the entire area of the modern Sahara, between the Nile, the Senegal and the Maghreb during tens of thousands of years. Its tools are well known, which tend to become smaller and lighter during the later phases in the Northwest (modern Morocco). Rigorously Paleolithic art is now known from this period in Upper Egypt, dating prior to 23,000 BP (Huyge 2013). Its stylistic patterns are identical to those of rare sites attributed to definitive Solutrean in Iberia, specifically when bifacial industries arrived as the "Middle Solutrean». The Proto-Solutrean phases grouped under this term in fact belong to Gravettian variants coming from Central Europe and were incorrectly interpreted. During its European expansion, the Solutrean creates a clear break in the prehistory of Europe. Limited in its extremely western part, it rapidly disappeared when the last Gravettians adopted ways of life and values leading to the expansion and development of the Magdalenian.

Keywords: Solutrean, Africa, nationalism, visual arts.

\section{LA MYOPIE NATIONALISTE}

Tandis que toutes les importantes civilisations furent considérées très simplement comme des mouvements expansionnistes irradiant à partir d'un puissant centre formateur - le Rubané provient de la plaine hongroise, le Gravettien des steppes orientales (Kozlowski 1986), I'Aurignacien des chaînes du Zagros (Delporte 1998), les Celtes d'Europe centrale (Kruta 1992), le Magdalénien du Sud-ouest européen
(Otte 2012) - le Solutréen subit une totale carence quant à sa genèse, ou, pire encore, d'interprétations incompréhensibles et en totale opposition avec les données radiométriques et technologiques. Le "proto-solutréen", d'origine septentrionale est beaucoup trop ancien par rapport au Solutréen classique. De toute évidence, ces aberrations furent dues à deux phénomènes idéologiques superposés, mais d'influences et d'effets rigoureusement identiques: la France de Breuil voulait tout voir venir d'elle-même (Breuil

(') Université de Liège. Place du XX Août 7. B-4000 Liège (Belgique). marcel.otte@ulg.ac.be. 
1912) et François Bordes voulut ignorer l'Espagne franquiste, comme l'Allemagne, vaincue et dont la langue elle-même lui était inconnue (Bordes 1968). Ainsi, durant un siècle, toute la reconnaissance d'une civilisation aussi fondamentale futelle mise en suspend, placée dans un nuage d'ignorance chaotique et impénétrable pour deux raisons: en France, on ne s'oppose pas aux mythes (ceux de Charles de Gaule ou comme ceux de François Bordes), en France on ne cherche pas ailleurs! Que les Gallo-Romains viennent de Rome, que les "Francs" soient une tribu germanique, que Charlemagne vienne de Liège, qu'importe (Otte et Keeley 1990).

\section{LES TIMIDES AUDACES}

Luis Péricot (1952) avait reçu l'ordre du "Général" Franco de ne rien voir de magrébin en Préhistoire espagnole et, en quelque manière, de ne jamais citer de connexion avec I'Afrique! Depuis Isabelle la Catholique, il fut proclamé que I'Islam et les Arabes devaient être tranchés du noble continent européen, oubliant le génie apporté en tous domaines, par les Juifs et les Arabes en lbérie et en provoquant ainsi la chute de l'Espagne au seul titre de son idéologie, une fois encore. Ce sort fut suivi par les Solutréens eux-mêmes, en dépit de dates plus anciennes en Espagne qu'en France (Jordá Cerdá 1976; Otte 1997), la phobie de l'Afrique combattait, surmontait, aveuglait contre toute évidence (Howe et Movius 1947; Otte et al. 2004).

\section{ARGUMENTS}

Considérons quelques-uns: La Pileta, El Parpalló, Bidon, par exemple s'opposent radicalement aux autres codes plastiques européens: il s'agit de silhouettes "découpées", dans le sens le plus strict de la tendance propre aux contours réalisés par nos enfants dans du carton: il n'existe rien autour et toute la "substance" de l'image tient en la matière propre au matériau découpé, à l'exact inverse de toute peinture pariétale où l'image s'intègre à la surface "décorée", à son volume, à son rythme, à ses proportions, à son grain (Fig. 1). Cette opposition apparaît d'autant plus nette lorsqu'on la compare aux œuvres stylistiquement analogues et réalisées dans les mêmes extrémités de la péninsule européenne: Lascaux, Le Gabillou, Pech-Merle, Cosquer, Pair-non-Pair par exemple où, justement, l'image fait corps avec la roche, autant par sa texture que par sa forme (Fig. 2). Depuis Altamira, nous sommes restés excessivement concentrés sur la seule existence de cet art, non sur ses modalités, un peu comme si on confondait encore l'art sur panneau avec l'art sur toile, ou les lois suivies par les fresques des cathédrales avec les vitraux qui s'y inscrivent! Cette confusion s'accentue encore lorsqu'elle s'inscrit dans la même région: l'art solutréen (Covalanas, Pasiega) ne possède aucune relation plastique avec celui de Tito Bustillo, Castillo, Santimamiñe par exemple.

\section{LES TECHNIQUES}

Une aussi radicale opposition plastique sépare les outils solutréens avec ceux qui l'encadrent, mais la myopie s'est étendue là aussi: tout ce qui fut rangé dans un opportun "Protosolutréen" complètement artificiel, n'est rien d'autre que le Gravettien d'Europe centrale (Otte et Noiret 2002) (Fig. 3). Mais il aurait fallu franchir la même frontière idéologique qu'avec l'Afrique et, accessoirement, savoir lire d'autres langues. Une simple observation des seules planches aurait même pu suffire, mais le dogme ne se limite pas aux messages écrits, il s'impose aux formes également. Lorsque Fr. Bordes avait si justement rejeté les pointes foliacées du Moustérien allemand de la genèse Solutréenne (1968), car beaucoup trop anciennes, il s'est fondé sur les textes déjà largement dépassés par la littérature allemande (Freund 1952). Mais il n'avait pas vu, par la même occasion, qu'il mettait en cause, sa propre définition de ses trop nombreux "faciès" moustériens: dès lors, le "Micoquien" n'existait plus, il ne s'agissait que d'une forme germanique du Moustérien.

Une simple observation portée sur la répartition $d u$ "Protosolutréen" eut clairement indiqué sa concentration vers l'Est et le Nord de l'Hexagone, c'est-à-dire, au Gravettien d'Europe centrale (Figs. 4 et 5).

\section{DATATIONS}

Exactement la même vision eut été aveuglante si elle s'était tournée vers la péninsule ibérique: toutes les dates du mythique stade moyen y sont plus anciennes qu'en France (Jordá Cerdá 1976).

Autre cas de cécité: toutes les dates, les données, les idées, joignant l'Andalousie au Maghreb furent ridiculisées, oubliées, méprisées: Bruce Howe (1947) fournissait toute la technologie identique à celle toute proche d'El Parpalló (Valencia) (Fig. 6). Les dates ${ }^{14} \mathrm{C}$ furent recalculées, 28000 BP (Debénath, 1986), identiquement que pour la "Grotte des Contrebandiers", 26000 BP. Rien n'y fit: le dogme l'emporte toujours sur les faits, car il terrorise.

\section{L'ATÉRIEN}

L'immense extension de l'Atérien, du Sénégal à l'Égypte et au Maghreb ne fut jamais considérée comme ce qu'elle fut: un territoire culturel, démographique, géographique et chronologique gigantesque dont l'issue ne fut pas même 


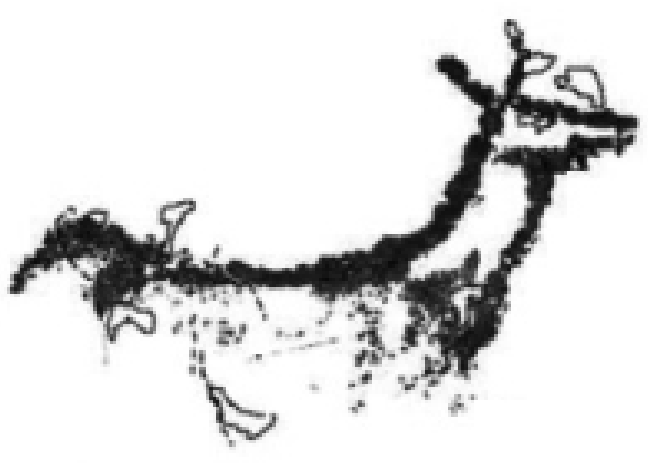

Coms de Arewaga
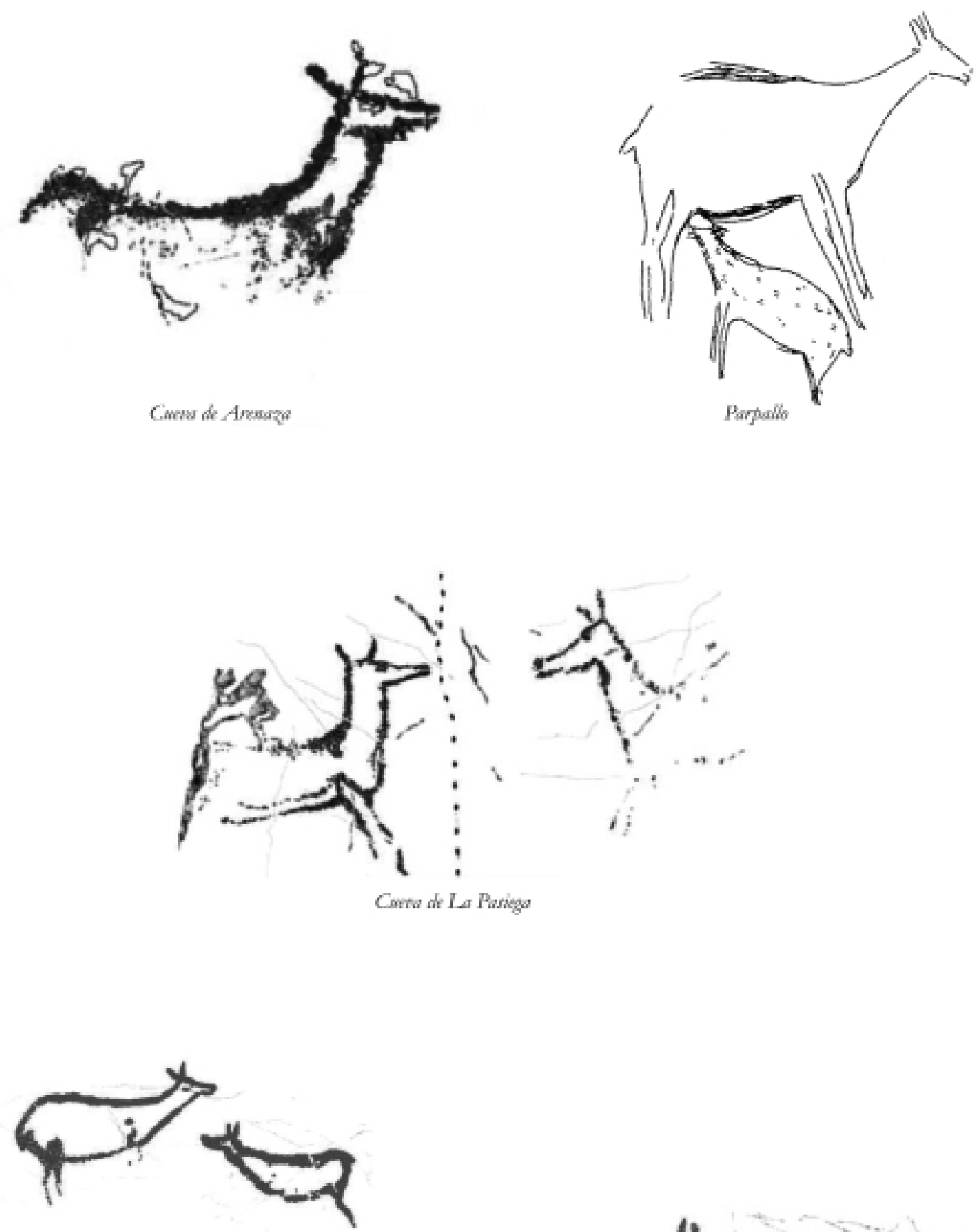

Comes \& Coushamas

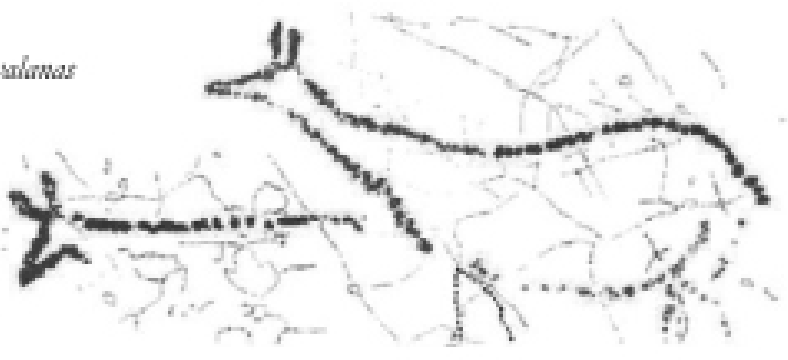

\ FiguRE 1. Iconisme restreint, relation à la nature, mise en scène et silhouettes découpées constituent les codes de l'art solutréen en Europe, telles des images, gravées et sculptées, en Afrique (Sources: Gárate Maidagan 2008; Otte 2012). 

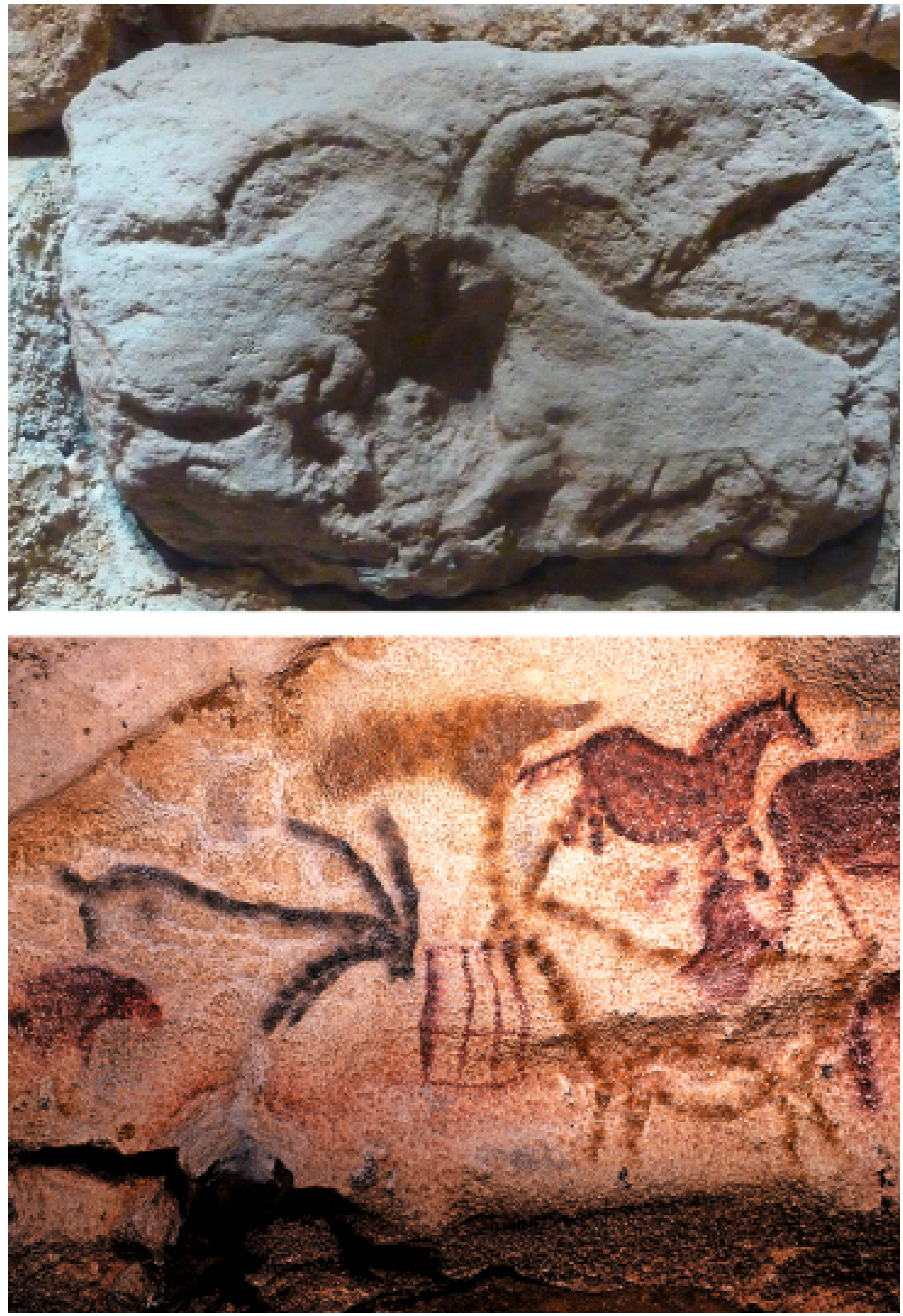

\ FiguRE 2. L'opposition frontale des bouquetins passe d'une analogie solutréenne (haut) au langage plastique hautement codifié au magdalénien (bas). Jeux de couleurs, de signes, de textures rendent l'image européenne créatrice de rêves, défiant la réalité (Sources: hanetcha-Parisienne.blogspot.be (Roc de Sers); linternaute.com/science/magazine (Lascaux)). 

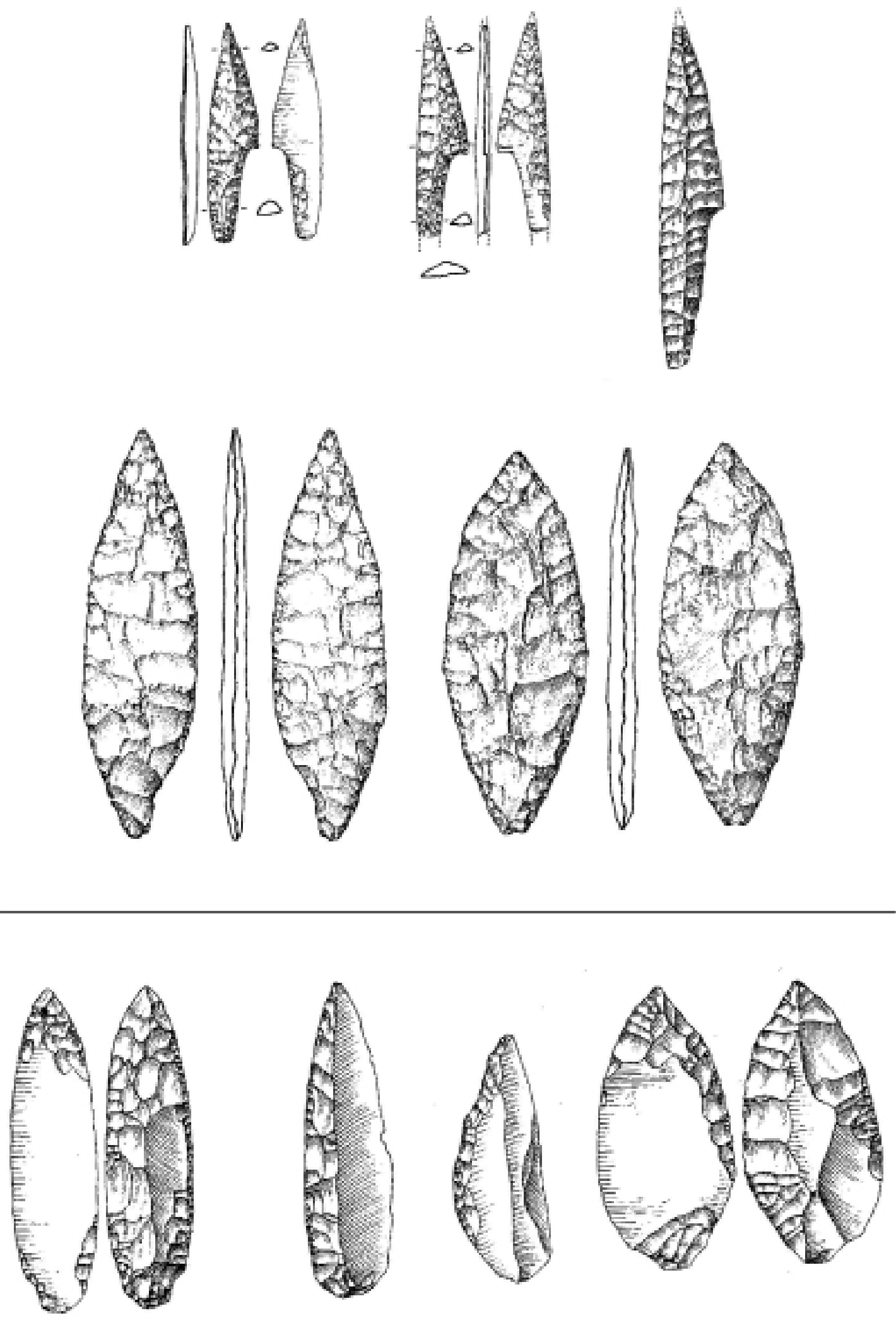

\ Figure 3. L'illusion de la continuité régionale, conforte le mythe de l'histoire nationale, et cette image l'illustre avec obstination et perversion. Toutes comparaisons extérieures à ce nombril culturel montrent l'influence de l'Est en bas, du Sud en haut (d'après Smith 1966, modifié). 


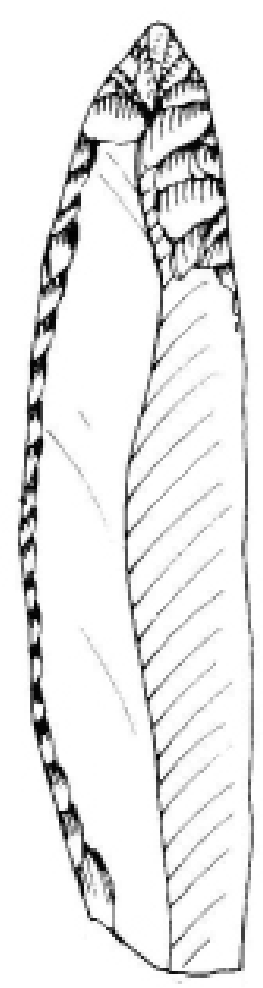

Molodora

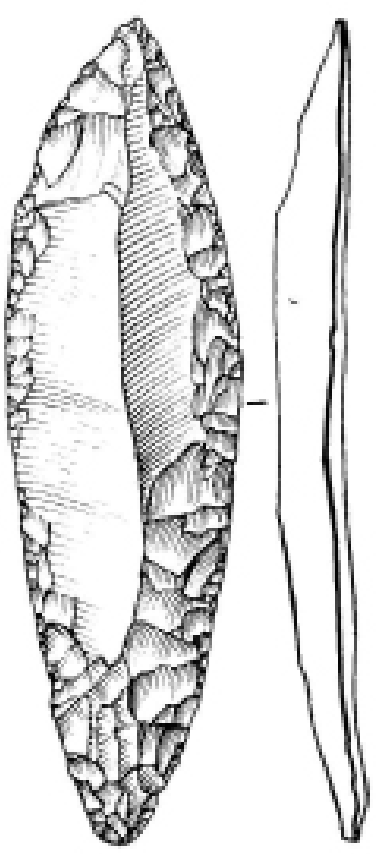

Laugerie-Hawte Onest

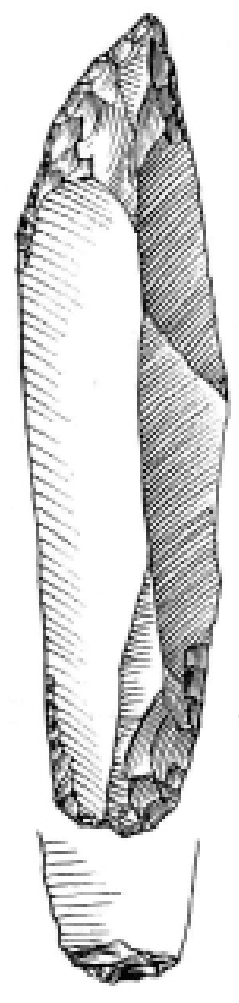

Langerie-Haste Est

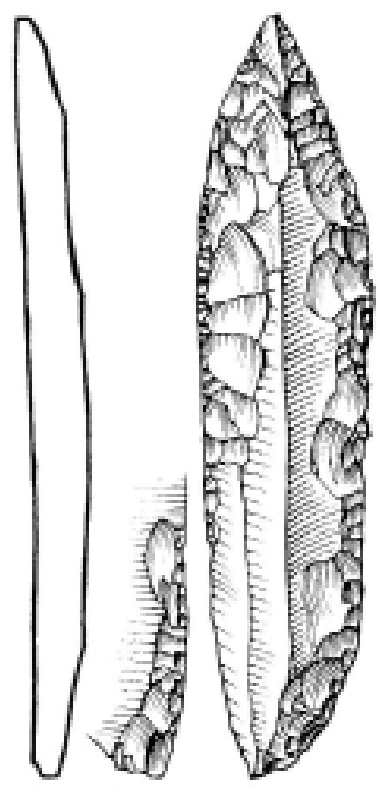

A FigURE 4. Les "lames appointées à retouches plates" du Gravettien oriental se métamorphosent, au Nord de la Loire, en "pointes à face plane", évidemment "Proto-Solutréennes" - La magie a fait le reste (Sources: Smith 1966; Otte 1981). 


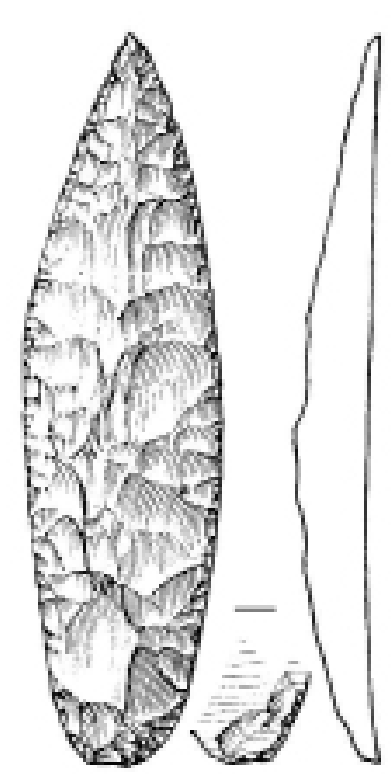

Lamgerie-Hawte Fat

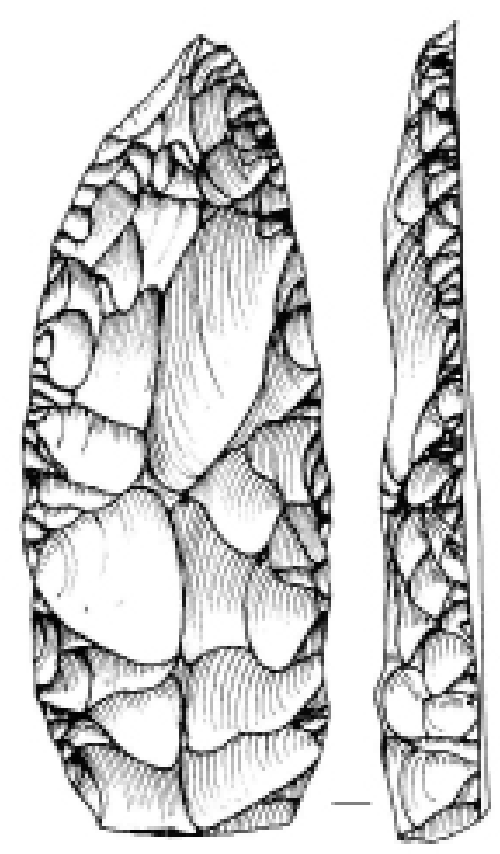

$S p y$

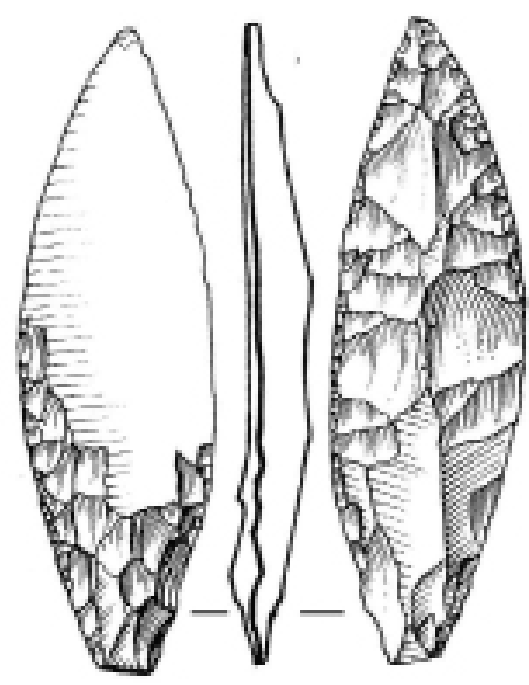

Langerie-Hawte Omest
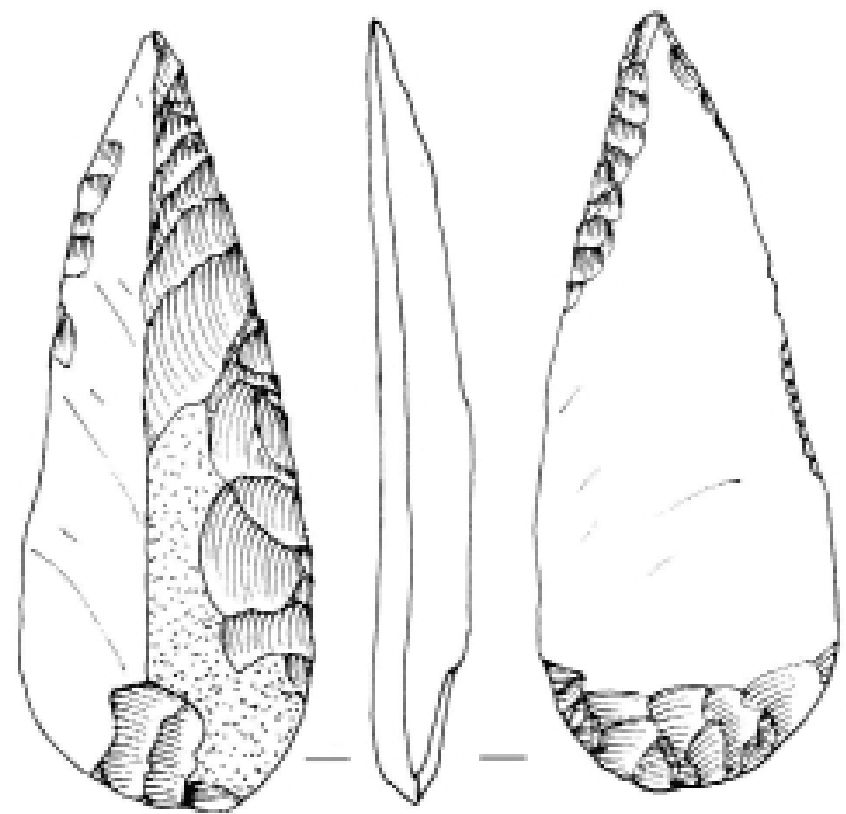

Willendorf

$\Delta$ Figure 5. Au cours de leur passage, de l'Ouest (en haut) à l'Est (en bas) de l'Europe, les objets identiques changent de sens, de nom, de rôle et de destin dans une littérature bien plus sédentaire que les peuples étudiés (Sources: Smith 1966; 0tte 1979). 
Parpallto (Vakescia)
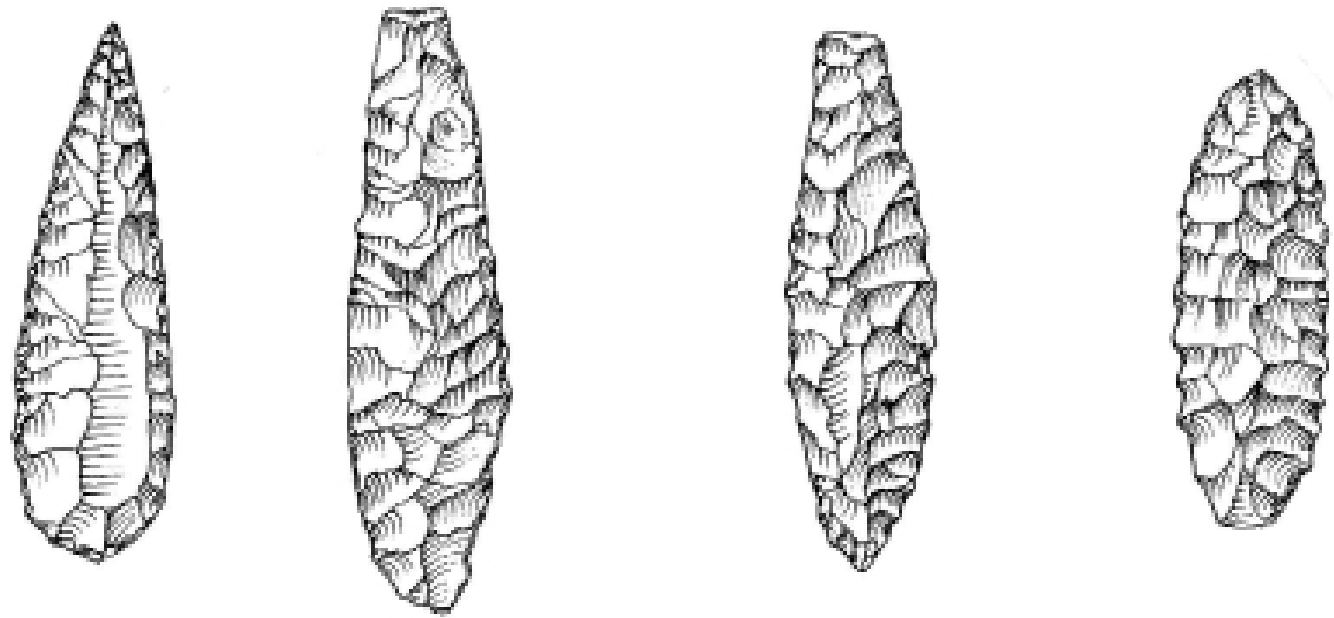

El Alya (Tanger)
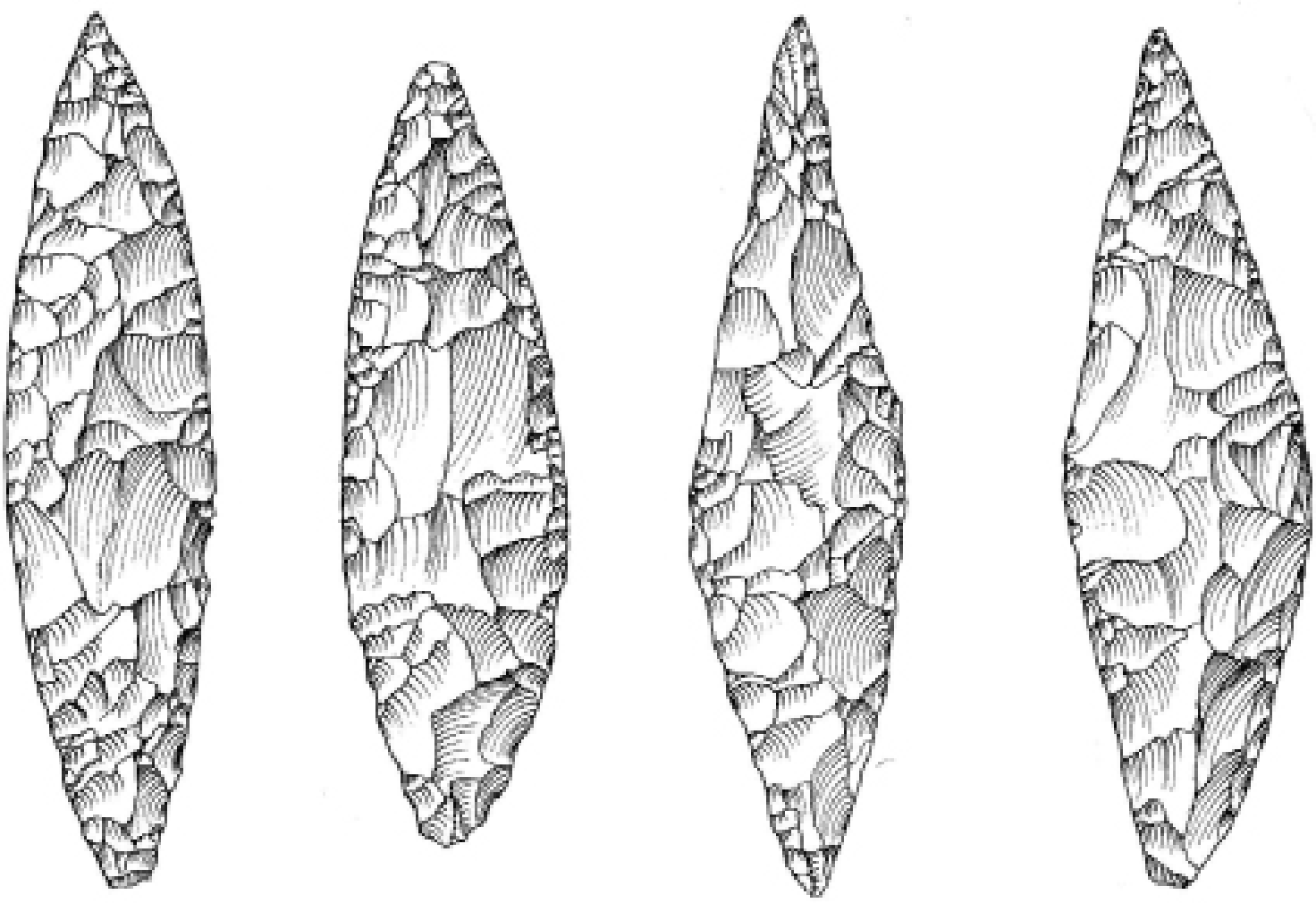

A FiguRE 6. L'analogie formelle, si puissamment évidente pour des objets aussi chargés de pratiques traditionnelles, n'ébranla pas le dogme, malgré les travaux pionniers de Bruce Howe et Hallam Movius (Source: Otte. et al. 2004). 


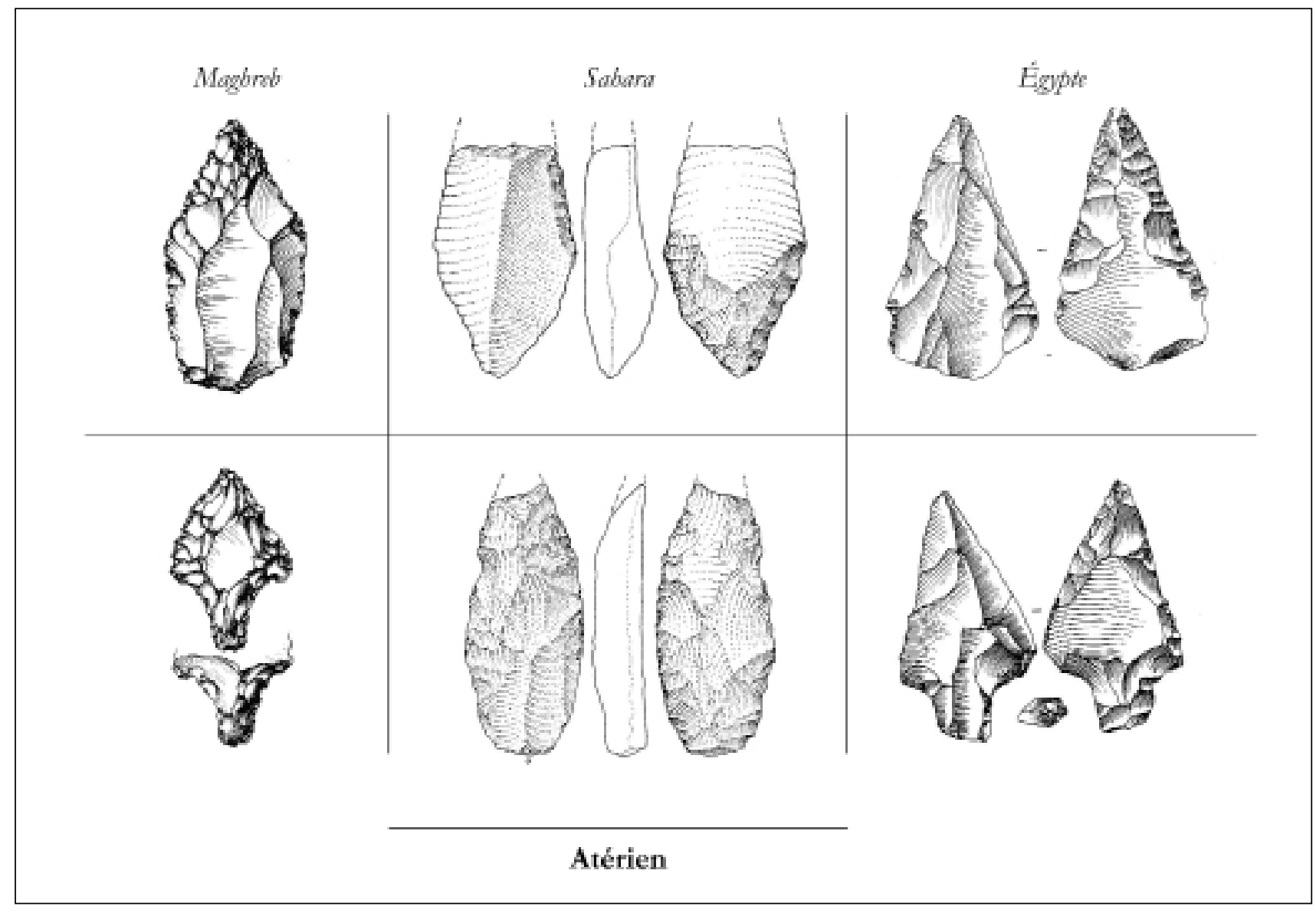

\ FIgURE 7. D'un bout à l'autre de l'Afrique du Nord, la civilisation atérienne impose son unité technique, plastique, anatomique, sans qu'aucun frémissement théorique ne vienne troubler les lois imposées par le terrorisme de certaines écoles (Sources: Camps 1974; Krzyzaniak. et al. 2000; Fiedler et al. 2003).

considérée quant à sa phase récente, à sa survie, à son prolongement vers la côte méditerranéenne (Fig. 7). Or, l'Europe était à deux pas, spécialement lors de l'abaissement général des niveaux océaniques, comme Jacques Collina-Girard (2009) n'a pas cessé de le répéter dans une série de publications aussi abondantes qu'ignorées (Fig. 8).

La même ignorance règne encore aujourd'hui quant aux découvertes d'art rupestres paléolithiques du Haut Nil (Huyge 2013). Aucun intérêt, aucune réaction, aucune conséquence européenne (Fig. 9). Seul, Emmanuel Guy (2012) en a tenté l'intégration dans toutes ses conséquences (2012), si brillamment même qu'elles furent aussitôt reléguées dans I'oubli. II a montré les lointaines connexions plastiques entre le Haut-Nil et le Sud de l'Europe.

\section{CONSTITUTION DU SOLUTRÉEN}

La nomenclature fut elle-même source d'une immense confusion!: un site au sud de la Bourgogne ne "pouvait pas" posséder une origine méridionale. Sa symbolique ellemême s'y opposait: il a suffi d'un rocher en surplomb, visible de l'autoroute, lieu d'ascension d'un Président en mal de réputation, d'une légende sur ses chevaux abattus: tout y était et y est encore pour confondre la dénomination "Solutréen" avec sa mythologie, très contemporaine et totalement fausse: car il s'agit d'un site "Périgordien" comme tous les Périgourdins se plaisent à le dire, soit "Gravettien" comme tous les autres peuples de la Terre désignent cette civilisation, elle aussi originaire d' "ailleurs", mais pas de là (Garrod 1938)! Bien que des traces d'occupation solutréenne y furent également observées. Solutré étant en France, le Solutréen en venait aussi...

Alors que tout désigne une orientation vers les outils, léger et bifaciaux, dans l'Atérien final du Maghreb septentrional, alors que les dates récentes y fourmillent les sites s'y concentrent, les îles surgissent (Collina-Girard 2009), I'art s'y concentre, rien n'y fait: le Solutréen c'est nous, l'Atérien ce sont eux! (Figs. 10 et 11).

La désertification s'accentue vers 20.000 ans, poussant les peuples du Sahara vers ses marges septentrionales. Lorsque l'outillage se conforme à la laminarité, nécessaire en Europe à l'emmanchement aux matières osseuses, encore rien.

Les souvenirs de l'Atérien, par retouches bifaciales, par pression, sur supports étroits, étendus à tous les supports 

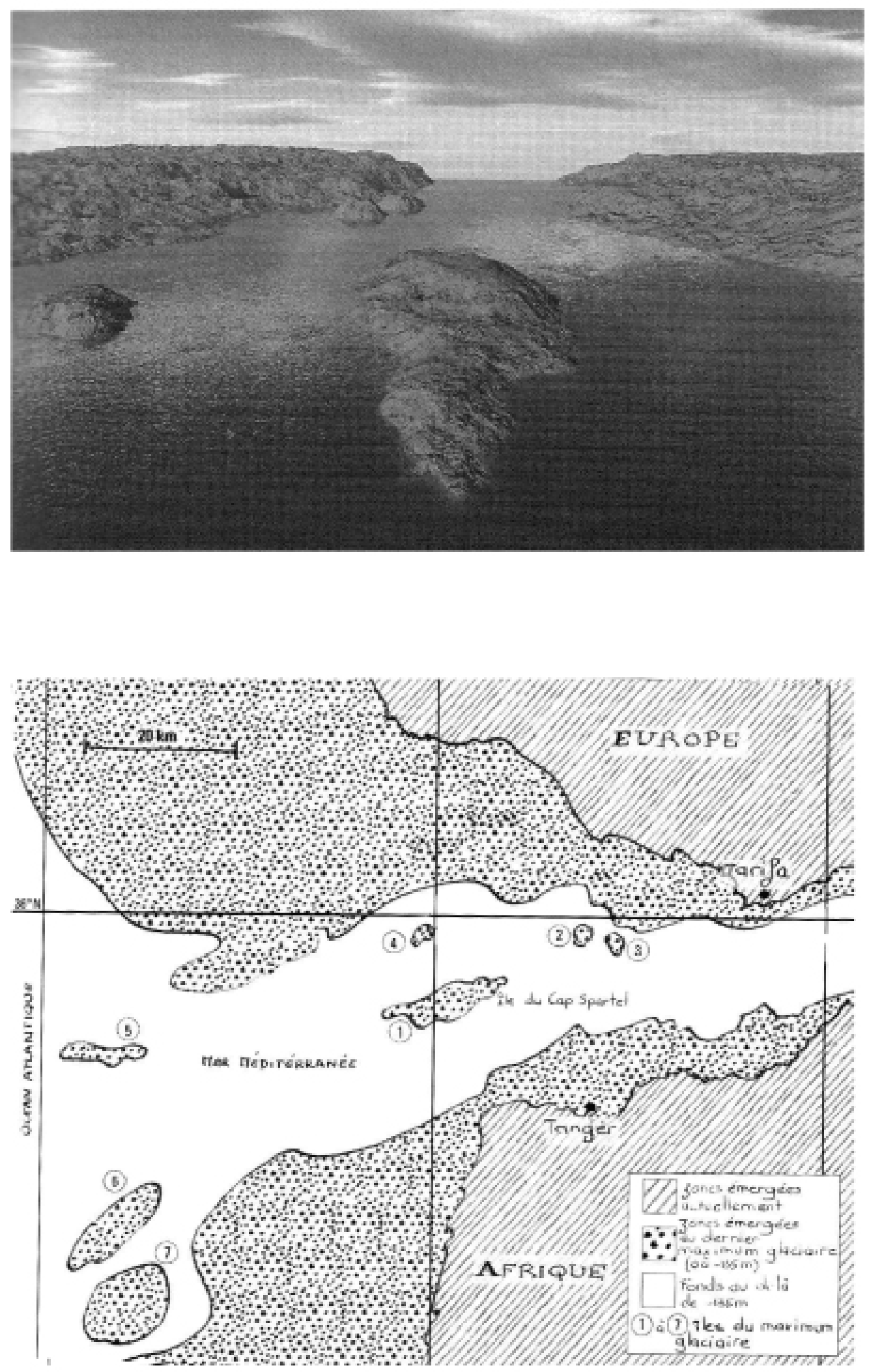

\ FiguRE 8. Traverser un bras marin n'a jamais posé le moindre problème à une population humaine (Australie, Polynésie). On pourrait même s'étonner que ce fût si rarement attesté. Mais, au pléniglaciaire, le surgissement d'archipels rend scandaleux le refus systématique de cette idée toute simple (Source: Collina-Girard 2009). 


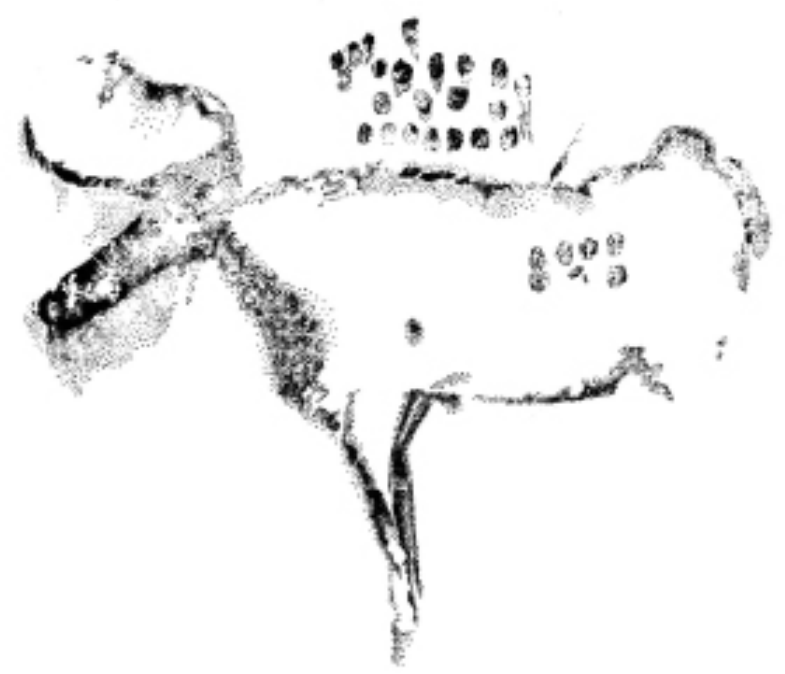

Bidon

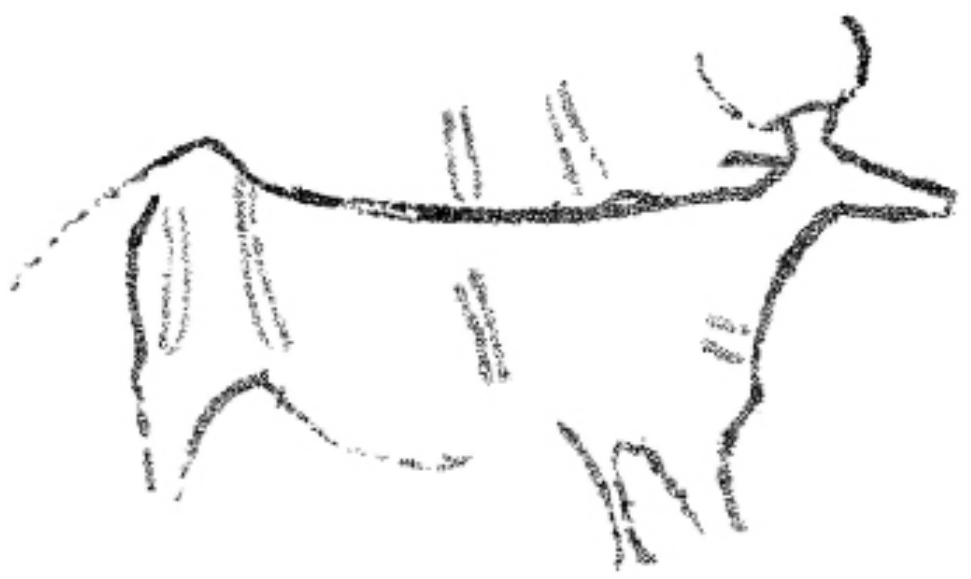

La Pileta

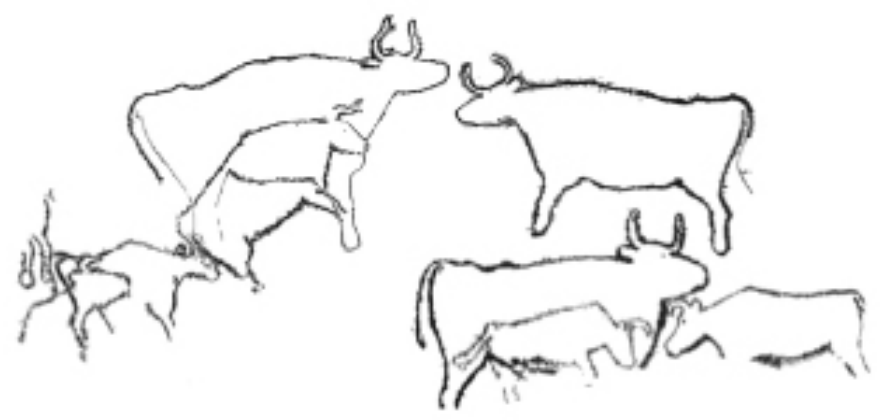

Qurta

\ FiguRE 9. Les lois plastiques solutréennes émergent aux deux extrémités de l'immense territoire atérien: du Nil à l'Andalousie (Sources: Guy 2012, d'après Huyge et al. 2011; Otte et Noiret 2002). 


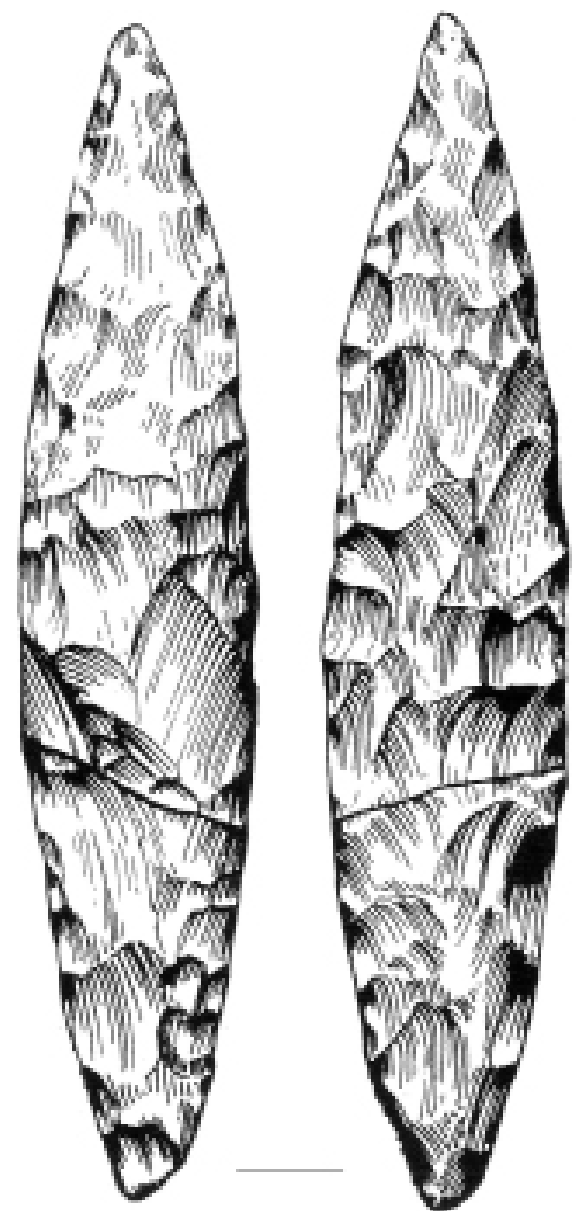

Adnar Baos site IV b (Teriri-Sahana awtral)

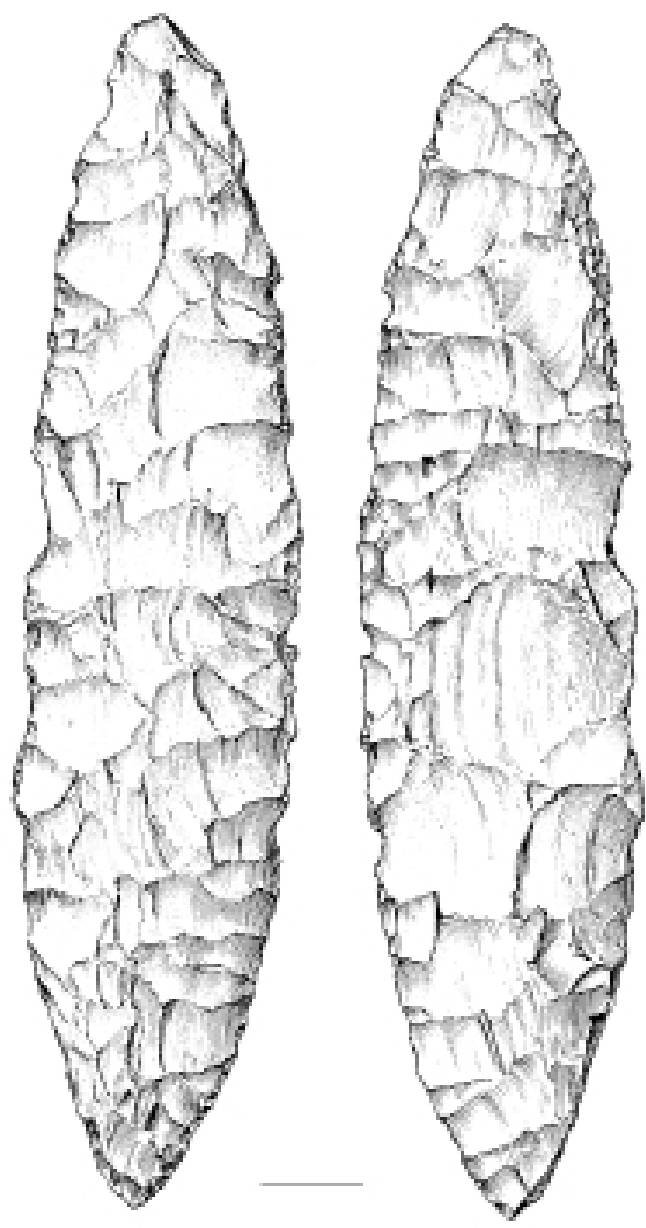

Les Jown-Bincs

\ FiguRE 10. Remise à la même échelle, les pointes sculptées bifaciales procèdent exactement de la même pensée des deux côtés du Détroit (Sources: Camps 1974; Smith 1966).

(Otte 1975) rien n'y fit. C'est à désespérer de la fécondité entre informations et imaginations, règle absolue en toute science, dont la Préhistoire semble vouloir malencontreusement se distinguer!

\section{LES ARTS}

Car toute discipline fonctionne, vit et progresse dans cette dualité, aussi impensables isolément qu'indispensables dans leur dynamisme (Kuhn 1973). L'imagination tire les données vers le haut et induit une théorie dominante et "créatrice de mythes" du Magdalénien, du Gravettien, de I'Aurignacien. Le rapport au mythe n'est pas exclu de l'art solutréen, il en est simplement décalé: son sort reste lié à la vision ressentie, elle-même chargée du pouvoir magique que les Européens paléolithiques croient trouver dans leurs rêves.

Si nous considérons la couleur, on peut dire qu'elle n' "existe" pas au Solutréen car sa fonction se réduit à délimiter deux aires: l'intérieur et l'extérieur d'une ligne, quelque soit leur teinte propre. Elle ne sort pas de son rôle de dessin de contours car ses fonctions d'à-plats, de modelés, d'harmonie chromatique lui échappent totalement en excluant toute sensibilité éventuellement issue de l'écho entre sa vibration et celle de l'âme. Son langage plastique semble directement dériver de la gravure sans relief, voire d'un contour découpé, à l'instar des roches plates ostensibles et sans mystère d'où elles proviennent, en Afrique.

Même les éléments mythologiques ("mythèmes") qui ont abusé les plus grands et de nombreux autres (sans citations) ont constitué des éléments de liaisons, mais en exacte 


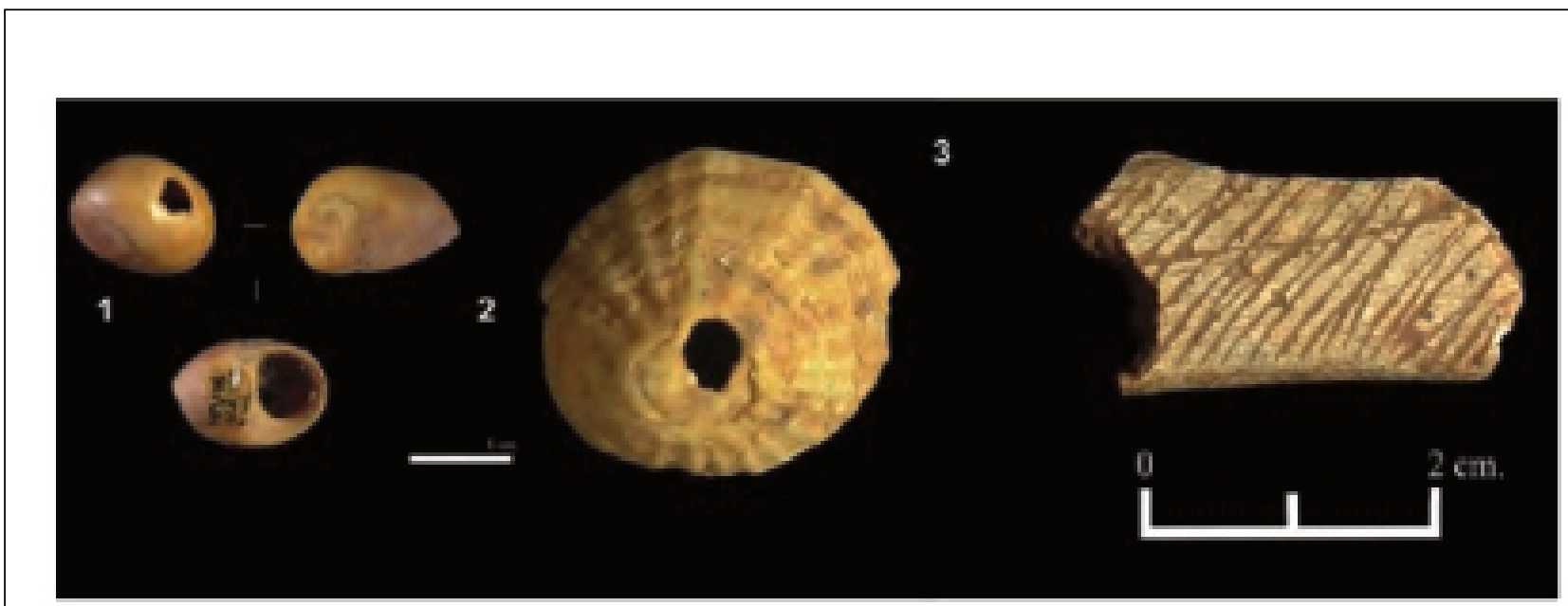

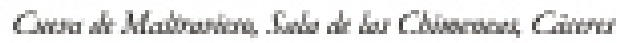

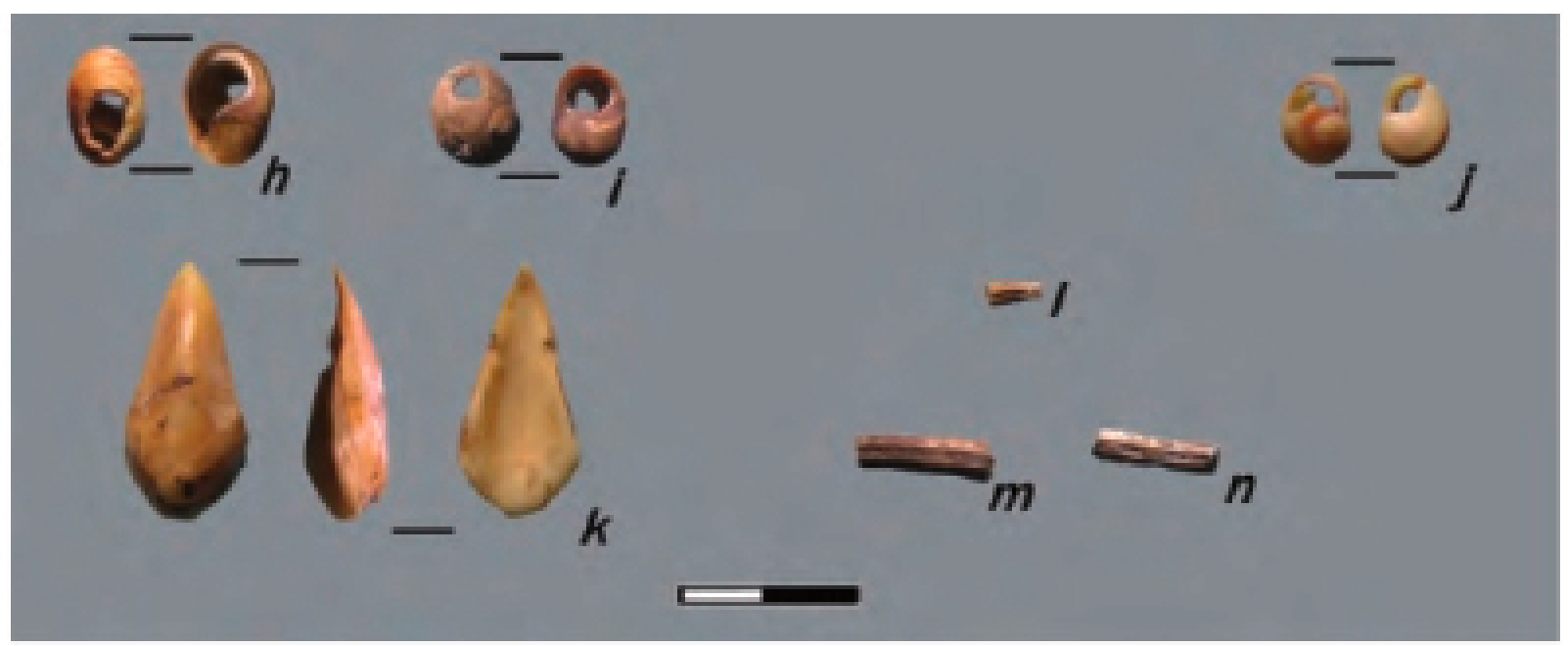

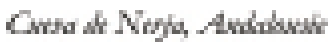
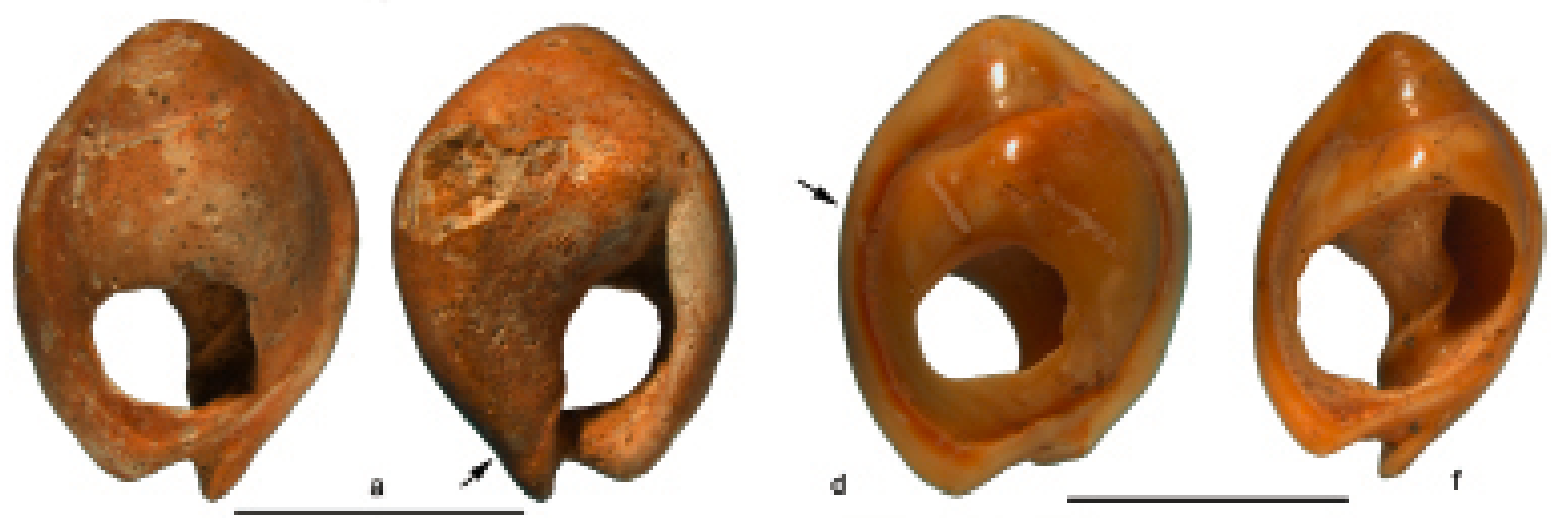

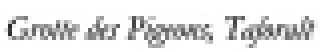

\ FIgURE 11. Rapports méditerranéens identiques quant aux coquilles perforées (Sources: Canals 2010; Aura et al. 2010; d'Errico et al. 2009). 
opposé à leur genèse propre: les bouquetins affrontés de Lascaux s'imprègnent d'une sémiotique élaborée, colorée et mystérieuse par l'intermédiaire des couleurs opposées, des signes intermédiaires, du rendu surréaliste de leurs profils (Fig. 2). Or, souvent, ceux tenus comme liaisons à Roc de Sers, se réduisent, eux, au réalisme le plus navrant: le modelé en relief sert à soutenir leur substance réelle, l'affrontement est fait d'un quotidien le plus banal, l'absence de couleurs leur confie une tonalité équivalente, pas de signe ésotérique amenant l'image à un langage extérieur, au vécu. Les roches elles-mêmes, dressées, séparées, exhibées n'on rien à faire dans le mystère d'une grotte où elles eussent été, en quelque sorte, suspendues de leur statut réel d'où elles seraient physiquement déconnectées. Tout l'art du Côa exprime de façon plus éclatante encore toutes ces évidences, spécialement à Fariseu où toutes les figurations précèdent le Magdalénien.

\section{ARTS ET ACTIONS}

Le domaine réputé "technique" représente en fait une forme de réponse entretenue entre les valeurs traditionnelles et les contraintes naturelles où l'habitat fut installé, il répond directement aux conceptions spirituelles qu'une société se fait d'elle-même, autant que l'art ou le mode d'échange alimentaire. Ainsi, celles du Solutréen reflètentelles la même pensée que celle, rendue visible par les codes plastiques. Or, ceux-ci s'expriment très clairement, en complète opposition aux arts produits pour les Européens déjà établis.

Selon cette façon de voir, les réponses dites "techniques" à l'environnement contiennent une partie des valeurs sacrées car elles fournissent les solutions mécaniques à la cause existentielle la plus forte de toute humanité: sa survie physique, dont toutes les autres dépendent. On n'abat pas un mouton de la même façon en islam ou dans la chrétienté, détail qui nous saute aux yeux mais qui devrait être multiplié par mille s'il s'agissait des traditions antérieures à notre banale quotidienneté. Or, tous les outils en pierre du Solutréen se reconnaissent au premier coup d'œil, autant par leur finesse, leur régularité, leurs retouches si régulières qu'elles transforment tout en œuvre d'art dans le sens le plus ordinaire de l'expression (Otte 1975). II n'est pas besoin d'être esthète pour ressentir que cette théorie masque les données nouvelles, alors nous nous réduisons à une humble ignorance... Les philosophes s'emparent joyeusement et sans scrupule et sans même de réactions articulées de notre élan. Si, naïvement, nous affirmions que tel art est solutréen car il est daté de 20000 ans, ils nous riraient au nez, en s'efforçant par courtoisie de cacher leur sourire. Inversement si nous argumentions par une analyse visuelle, pourquoi cette œuvre se distingue des codes magdaléniens, alors ce philosophe rentrerait son sourire dans sa barbe (car ils en ont souvent) et chercherait d'abord à suivre le raisonnement et ses yeux s'embraseraient par vos explications.

\section{PLASTIQUE}

Alors, pourquoi la Pasiega est-elle solutréenne bien que juste au-dessus d'elle, Las Chimeneas, Las Monedas et le Castillo ne le sont pas? Très simple: il existe un langage plastique aussi cohérent que celui que vous lisez à l'instant: vous ne le confondrez jamais avec une autre langue, même si elle est écrite avec les mêmes caractères latins ! Reprenons l'image solutréenne: elle est encore plus simple à déchiffrer car son iconographie (son "alphabet") s'oppose à celui du chinois, pour poursuivre cette délicate métaphore... Les biches d'El Parpalló et de la Pasiéga sont rares dans les autres "langues" paléolithiques. Et les liaisons grammaticales ne s'y conforment pas davantage: leur cou est tendu, il s'anime comme dans la réalité, il observe, il suit, tel un réel troupeau. Cette liaison syntaxique proche de l'observation, comme une photographie peut l'être, fut totalement abandonnée par les autres arts, l'immédiate harmonie si bien dégagée par les " pointes solutréennes " qu'elles n'ont pu qu'être réalisées dans une perspective extérieure à leur efficacité technique ! La retouche bifaciale ne présente aucun intérêt, autre que sa sculpture symétrique, harmonieuse, sensuelle. L'efficacité n'a rien à y faire, pas davantage que les pointes de Clovis (USA), les haches a polissage bifacial (Scandinavie) ou les sabres japonais, autant façonnés pour leur beauté formelle (telle que précisément l'illusion de leur efficacité) que pour leur réelle utilité. Et tout va ainsi dans les sociétés raffinées, du charpentier à la potière: la laideur ou le mauvais goût n'y seraient acceptés que s'ils répondaient à des idées adéquates aux besoins qui y ont toujours dérangé l'esprit coutumier. Les découvertes d'outils solutréens élaborés rassemblés dans des poches enfouies montrent bien l'exceptionnel prestige accordé par la société aux artisans capables de les réaliser, telle une marque identitaire. Toute cette démarche n'a rien à voir avec l'outil contemporain européen reconnu pour sa seule efficacité: les statuts pris par les uns et par les autres démontrent clairement le rôle accordé aux objets utilitaires par l'Europe à autre chose. Comme cette civilisation se trouve strictement limitée à l'extrême sud-ouest, cet "autre monde" ne peut être que l'Afrique où l'extrême raffinement accordé à l'outillage caractérise toutes les civilisations, dès l'Atérien inclus, jusqu'à vingt mille ans où elle en disparaîtrait magiquement.

\section{OUTILS ET BEAUTÉ}

Glissée entre la résistance mécanique opposée par le monde naturel et les actions combinées qui les surmontent, 

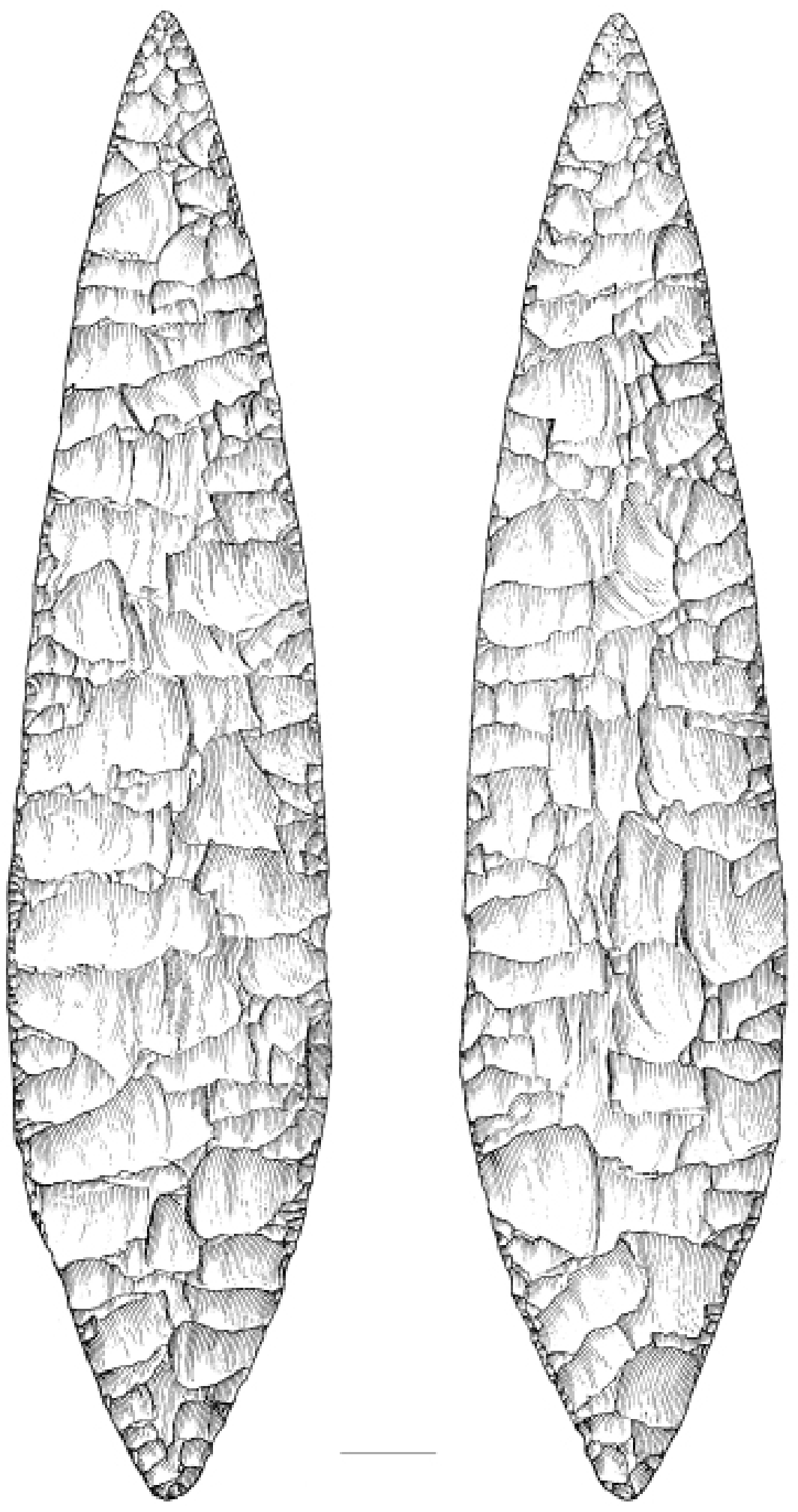

Pedj de la Boissière

\ Figure 12. La beauté plastique d'armes, inutiles et symboliques, marque le Solutréen (Source: Smith 1966). 


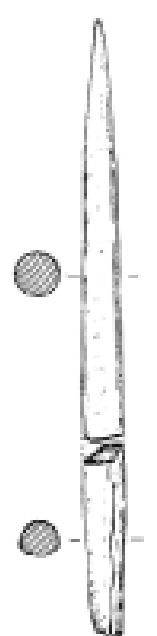

Las Caldas

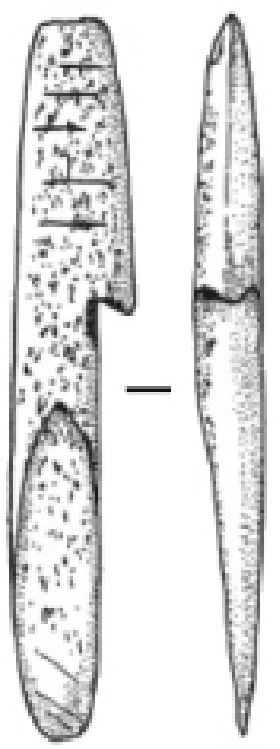

Grotfe du Placard

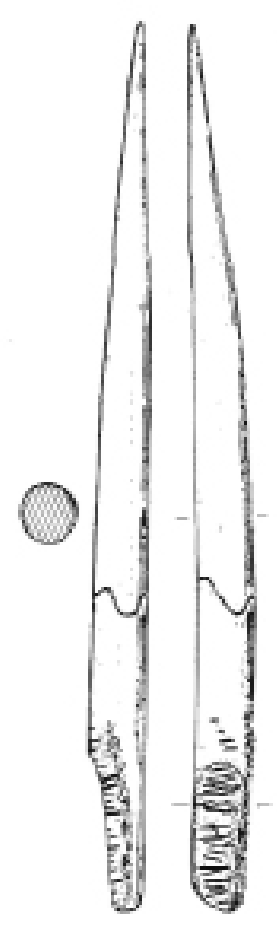

Creto de la Minda

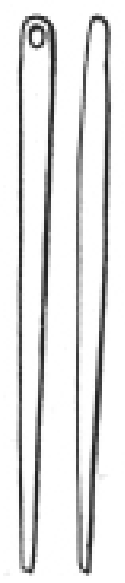

Pect de la Boissic̀n

\ FiguRE 13. La complexité des matières osseuses atteste cette nouvelle emprise dans tous les procédés (Sources: Cattelain 1989; Smith 1966). 
l'esthétique de l'outil active la corrélation entre les deux: elle ne les harmonise pas seulement, elle leur en donne une direction évolutive. Ainsi, les civilisations se transformentelles par le flux subtil de telles interrelations. Au sommet des créations symboliques totalement détachées de toute fonction technique, les outils solutréens acquièrent le seul statut de purs symboles, comme leur art l'était: inscrits dans une volonté active, ils en deviennent des témoins désacralisés, mais toujours ils incarnent d'identiques tentatives menées contre le réel (Fig. 12). Mais ces différentes formes d'expression se trouvent liées par un filet de pensées, d'autant plus serré que cette civilisation fut courte et délimitée spatialement. La Rome impériale absorbait les coutumes étalées des marges de l'Écosse à celles de I'Iran, tout en restant " romaines " durant des siècles. À l'échelle paléolithique, le Solutréen n'est qu'un flash, court et marginal, donc cohérent, comme il apparaît dans toutes ses activités.

\section{CODES TECHNIQUES}

Bien au-delà de tout ce réseau d'activités plastiques ou techniques, cette civilisation fit apparaître l'aiguille à chas et ses déferlements d'expressions vestimentaires où s'alignent le rang, le rôle, l'âge et la biographie. Très certainement rencontrées par d'autres biais auparavant, ces fonctions essentielles s'y trouvent tout à coup réunies et modifiées par la seule voie d'un instrument simple mais où s'agencent de multiples actions techniques: on peut alors parler de génie solutréen, jusque dans ses plus humbles expressions, poursuivies aujourd'hui dans sa plus totale uniformité. La tête du propulseur amovible fut apportée par la même réflexion, appliquée à une fonction tout autre, comme si cette pensée cohérente tenait à s'appliquer à de tout autres actions mécaniques. II ne fait aucun doute que le propulseur fut adopté par les premières populations nomades débordant de l'Asie vers l'Europe ; il n'est pas moins douteux que son usage se perpétua jusqu'en différents points du globe, telle une fulgurante convergence. Mais le génie solutréen en fit une arme articulée et composite, dont le coude allongeait le bras mais dont le crochet, où se concentrait la plus intense friction, pouvait ainsi être remplacé, sans perdre le reste de ses autres éléments mécaniques où se place autant l'embouchure de la hampe, les préhampes au sommet du fût la pointe elle-même et le bras de levier qui augmente la force propulsive. Par leur décomposition en éléments séparés, amovibles et interchangeables, le propulseur solutréen gagnait en souplesse, en durée et en efficacité (Fig. 13). Pour un peuple chasseur, cette augmentation subite de l'emprise nutritionnelle ne fut pas sans rapport avec la fierté très légitime qu'il en concevait, autant par rapport aux autres peuples mis en contacts et, surtout, par rapport aux forces naturelles qu'il maîtrisait, tel un créateur au profit de sa seule société dont les liens s'en trouvaient renforcés. Une telle humanité ne pouvait qu'être homogène et solidaire et c'est ainsi qu'elle peut-être reconnue, vingt mille ans plus tard, sans l'ombre d'une hésitation, tant qu'une misérable datation ${ }^{14} \mathrm{C}$ n'en vienne perturber l'évidente cohérence.

\section{LA TRADITION ET SON DESTIN}

II nous appartient donc d'imaginer une vaste communauté, démographique et culturelle, centrée sur le Sahara actuel et étendue entre le Nil, le Sénégal et le Maghreb. Toutes les données techniques témoignant de cette unité, globalement désignée d'Atérien, mais dont les œuvres d'art, curieusement analogues à l'Europe, viennent d'être reconnues en Haute-Égypte dans une phase antérieure à 23000 BP (Huyge 2013). Dans l'extrémité nord-occidentale, les grottes d'El Alya, des Contrebandiers et d'Ifri N'amar, affirment cette extension, par un Atérien récent, aux pièces bifaciales légères, identiques à celles du Solutréen ibérique. Une sorte de cécité, politiquement entretenue, en a interdit la clairvoyance. D'autant plus évidente que les grottes andalouses, distinctes et isolées du reste de l'Europe, en constituaient le relais artistique tout proche. Ses codes plastiques s'étendent jusqu'au Côa au Portugal dans ses phases récentes, à El Parpalló en Méditerranée, à Covalanas en Cantabrie, ils pénètrent jusqu'en plein Périgord, au Roc de Sers, où les blocs monumentaux sont ostentatoires, comme en Afrique. Cette civilisation a pu subsister tant que les liens et les valeurs restèrent alimentés par un flux continu. Mais l'absence de toute acculturation illustre clairement sa puissante cohésion. Sa disparition subite en constitue une autre preuve: le Gravettien se poursuit en parallèle dans tout le reste de l'Europe. Sa résistance, idéologique et technique, aux sources du Magdalénien régional, montre à l'inverse la puissance des peuples qui ont entouré, défié puis submergé le Solutréen. Sur une carte chronologique de l'Europe, on voit clairement la persistance des flux gravettiens alimentés tout au long de l'Eurasie. Tout semble indiquer que ce flux migratoire avait cessé avec l'Afrique dès que les détroits furent impraticables et que l'Atérien, lui-même, disparut dans l'histoire de l'Afrique, pour aucune autre raison logique que l'arrivée, le long du littoral magrébin des peuples dits "Ibéro-Maurusien" à technologie beaucoup plus élaborée et aux arts introduisant enfin des "scènes" où l'homme apparaît en coordinateur. Exactement le même mouvement idéologique progressa bientôt dans les tentatives européennes pour maîtriser le destin des peuples vers l'attitude moderne, aux confins du Néolithique. • 


\section{BIBLIOGRAPHIE}

Alcaraz Castaño, M. 2007: "El Ateriense del Norte de África y el Solutrense peninsular: ¿contactos transgibraltareños en el Pleistoceno Superior?". MUNIBE 58: 101-126.

AntoINE, M. 1952: Les grandes lignes de la Préhistoire marocaine, lle congrès panafricain et Préhistoire. Les impressions Édita. Alger.

Aura, J. E., JordÁ, J. F., Pérez, M., Badal, E., Morales, J. V., Avezuela, B., TIFFAGOM, M. et JARDÓN, P. 2010: "Treinta años de investigación sobre el Paleolítico superiorde Andalucia: la Cueva de Nerja (Málaga, España)". En X. Mangado (ed.): El Paleolitico superior peninsular. Novedades del siglo XXI. SERP, Universitat de Barcelona, Barcelona: 149-172.

Barton, R. N. E., Bouzouggar, S. N., Collcutt, J. L., Schwenninger, L. et Clark-BAlzan, L. 2009: "OSL dating of Aterian levels at Dar es-Soltan I (Rabat, Marocco) and implications for the dispersal of Homo sapiens". Quaternary Science Reviews 28 (19-20): 1914-1931.

Belting, H. 2004: Pour une anthropologie des images. Éditions Gallimard. Paris.

BoRDES, FR. 1968: Le Paléolithique dans le monde. Paris. Hachette.

BosINSKI, G. 1967: Die Mittelpaläolithischen Funde im westlichen MieIleuropa. Böhlau Verlag Köln Graz.

Bouzouggar, A., Barton, N., Vanhaeren, M., D'errico, F., Collcutr, S., Higham, T., Hodge, E., Parfit, S., Rhodes, E., Schwenninger, J.-L., Stringer, C., Turner, E., Ward, S., MoutmiR, A. et Stamboul, A. 2007: "82,000-yearold shell beads from North Africa and implications for the origins of modern human behavior". PNAS 104 (24): 9964-9969.

BREUIL, H. 1912: Les subdivisions du Paléolithique supérieur et leur signification. C.I.A.A.P. 14. Genève I: 126-129.

CAmPs, G. 1974: Les civilisations préhistoriques de l'Afrique du Nord et du Sahara. Éditions Doin. Paris.

Canals, A., Rodriguez-Hidalgo, A., Peña, L., Mancha, E., García-Diez, M., Bañuls, S., Euba, I., López-Garcia, J. M., Barrero, N., Bermejo, L., Garcia, F. J., Mejias, D., Modesto, M., Morcillo, A., Aranda, V. et Carbonell, E. 2010: "Nuevas aportaciones al Paleolitico superior del suroeste peninsular: la cueva de Maltravieso, más allá del santuario extremeño de las manos". En X. Mangado (ed.): El Paleolítico superior peninsular. Novedades del siglo XXI. SERP, Universitat de Barcelona, Barcelona: 199-218.

CATTELAIN, P. 1989: "Un crochet de propulseur solutréen de la grotte de Combe-Saunière (Dordogne)". B. Société Préhistorique Française 86 (7): 213-216.

ColuINA-GIRARD, J. 2009: L'Atlantide retrouvée ? Enquête scientifique autour d'un mythe. Éditions Belin. Pour la science. Paris.

De LA RASILLA VIVES, M. 1994: "Introducción: el Solutrense en el contexto del Paleolítico Superior Occidental". Férvedes 1: 9-19.

DEBÉNATH, A. 1992: "Hommes et cultures matérielles de l'Atérien marocain". L'Anthropologie 96 (4): 711-720.

Debénath, A., RAYNAL, J-P., ROChE, J., TeXIeR, J-P. et Ferembach, D. 1896: "Stratigraphie, Habitat, Typologie et devenir de l'Atérien marocain: Données récentes". L'Anthropologie 90 (2): 233-246.

DELPORTE, H. 1998: "Les Aurignaciens premiers hommes modernes". Histoire de la France préhistorique de -40000 à -25000 . Éditions La maison des roches. Paris.

D'Errico, F., Vanhaeren, M., Barton, N., Bouzouggar, A., Mienis, H., Richter, D., Hublin, J.-J., McPerRron, S. P. et Lozouet, P. 2009: "Additional evidence on the use of personal ornaments in the Middle Paleolithic of North Africa". Proceedings of the National Academy of Sciences of the United States of America 106 (38): 16051-16056.

ELoI, L. 1956: Le Proto Solutréen dans le bassin de la Meuse en Belgique. Le Mans.

FiedLeR, L., LUTZ, G., LUTZ, R. et QUeHL, H. 2003: Siedlungshistorische und paläoethnologische Untersuchungen im Wadi Tidoua, MessakMellet, Zentralsahara. Behausungsstruktur, Artefaktverteilung und Geräteformen eines atérienzeitlichen Fundplatzes. Römisch-Germa- nisches Zentralmuseum Forschungsinstitut für vor- und Frühgeschichte.

Freund, G. 1952: Die Blattspitzen des Paäolithikums in Europa. QuartärBibliothek Bd. 1. Bonn.

FrobenIuS, L. 1952: Histoire de la civilisation africaine, traduit de I'allemand par Dr Back, H. et Ermont, D. Éditions Gallimard. Paris.

GÁrate MaidaGAN, D. 2008: "Las pinturas zoomorfas punteadas del Paleolítico Superior cantábrico: hacia una cronología dilatada de una tradición gráfica homogénea / Les peintures animales cantabriques à tracé ponctué: vers une chronologie large d'une tradition graphique homogène". Trabajos de Prehistoria 65 (2): 29-47.

GARROD, D. 1938: "The Upper Palaeolithic in the light of recent discovery." Proceedings of Prehistoric Society 4: 1-26.

GuY, E. 2012: L'art paléolithique européen de la Vallée du Nil. Publié en ligne le $7 / 6 / 2012$. paleoesthetique.com

HowE, B., L. et Movius, H., JR. 1947: A stone age cave site in Tangier, Peabody Museum of American Archaeology and ethnology. Harvard University. vol. XXVIII. Nr 1.

HowE, B. 1967: The Palaeolithic of Gangier, Morocco. American school of prehistoric research Peabody museum. Harvard University. Bulletin No. 22. Éditions Lloyd Cabot Briggs and Hugh Hencken. Cambridge. Massachusetts. USA.

HuYGE, D. 2013: "Ice Age Art at Qurta". Ancient Egypt Magazine 13 (5) Issue 77: 32-41.

Huyge, D., Vandenberghe, D. A. G., De Dapper, M., Mees, M., Claes, W. et DarnelL, J. C. 2011: "First evidence of Pleistocene rock art in North Africa: securing the age of the Qurta petroglyphs (Egypt) through OSL dating". Antiquity 85 (330). The Free Library (December, 1), http://www.thefreelibrary.com/First evidence of Pleistocene rock art in North Africa: securing the...-a0276633781.

Jordá Cerdá, F. et Fortea Pérez, J. 1976: "El Paleolítico superior y Epipaleolítico mediterráneo español en el cuadro del Mediterráneo occidental". En D. Camps (dir.): Chronologie et synchronisme dans la Préhistoire circum-mediterranéenne. Union Internationale des Sciences Préhistoriques et Protohistoriques, IXe congrès. Nice: 99-127.

Kozlowskı J. K. (dir.) 1993: Atlas du Néolithique européen. Vol. 1: L'Europe orientale. ERAUL 45. Liège.

- 1986: "The Gravettian in Central and Eastern Europe". Advances in world Archaeology 5: 131-200.

KRUTA, V. 1992: L'Europe des origines. Éditions Gallimard. Paris.

KrzYZANIAK, L., KRoEPer, K. et Kobusiewicz, M. 2000: Recent Research Into the Stone Age of Northeastern Africa. Pozna'n Archaeological Museum. Pozna'n.

KuHN, TH. 1973: La Révolution Copernicienne. Éditions Fayard. Paris.

OTтE, M. 1975: "Documents solutréens de France conservés au musée Curtius à Liège". Bulletin de I'Institut Archéologique Liégeois. t. LXXXVII. Liège: 79-95.

- 1979: Le Paléolithique supérieur ancien en Belgique. Bruxelles. Monographies d'Archéologie Nationale. Vol. 5.

- 1981: Le gravettien en Europe centrale. Dissertationes Archaeologicae Gandenses XX. Bruges.

- 1997: "Contacts trans-méditerranéens au Paléolithique". En J. M. Fullola et N. Soler (éd.): El Món Mediterrani Després Del Pleniglacial (18.000-12.000 BP). Actes du colloque de Banyoles (18-20 mai 1995). Girona, Musée d'Archéologie de catalogne. Sèrie Monographica 17: 29-39.

- 2012: "Appearance, expansion and dilution of the Magdalenian civilization". Quaternary International 272-273: 354-361.

0TE, M., Bouzouggar, A. et Kozlowskı, J. K. (dir.) 2004: La Préhistoire de Tanger (Maroc). ERAUL 105. Liège.

OTE, M. et KeELEY, L. 1990: "The Impact of Regionalism on Palaeolithic Studies". Current Anthropolog. 31 (5): 577-582.

OTE, M. et NolRet, P. 2002: "Origine du Solutréen: le rôle de l'Espagne". Zephyrvs 55: 77-83. 
Peregrine, P. et Ember, M. (eds.) 2001: Encyclopedia of Prehistory (4): Europe. Kluwer Academic/Plenum Publishers. New York: 328-350.

PeRICOT, L. 1942: La Cueva del Parpalló. Madrid: CSIC/N.

- 1949: "Review of "A Stone Age Cave Site in Tangier" (Howe and Movius, 1947) and "The Prehistoric Archaeology of the Tangier Zone, Morocco" (Hencken, 1948)". Ampurias 11: 22-226.

- 1952: "Sur les connexions européennes possibles de l'Atérien. État actuel du problème". Actes du lle Congrès panafricain de Préhistoire. Alger: 375.

- 1953: "Sobre el problema de las relaciones preneolíticas entre España y Marruecos". I Congresso Arqueologico des Marruecos Español (Testuá, 1953): 57-65.

RIPOLL LóPEZ, S. 1989: "Le gisement de la Cueva de Ambrosio: nouveaux apports au solutréen de la Péninsule Ibérique". L'Anthropologie 92 (4): 851-886.

SICARD, M. 1998: La Fabrique du Regard. Odile Jacob. Paris.
SMITH, P. E. L. 1966: Le solutréen en France. Publications de I'Institut de Préhistoire de l'Université de Bordeaux. Mémoire nr 5. Bordeaux.

SONNEVILLE-BORDES, D. 1959: "Problèmes généraux du Paléolithique supérieur dans le Sud-Ouest de la France". L'Anthropologie 63 (1-2): 1-36.

Straus, L. G. et González, M. R. M. 2009: "A preliminary description of Solutrean occupations in El Mirón cave (Ramales de la Victoria, Cantabria)". MUNIBE 60: 117-137.

TeXIER, J.-P., HuXtABLE, J., RHODES, E., MIALLIER, D. et OUsmOI, M. 1988: "Nouvelles données sur la situation chronologique de l'Atérien du Maroc et leurs implications". C. R. Acad. Sci. París 307 (Série II): 827-832.

TIFFAGom, M. 2006: De la Pierre à l'Homme. ERAUL 113. Liège.

UtrilLA, P. et MAZO, C. 1993: "Le Paléolithique supérieur dans le versant Sud des Pyrénées. Communications et influences avec le monde pyrénéen français". 118e Congrès National des sociétés historiques et scientifiques. Pau: 243-262. 
